\title{
The impact of comorbidities on the severity of atopic dermatitis in children
}

\author{
Vojko Berce ${ }^{1}$, Teja Marhold ${ }^{2}$, Vid Delopst ${ }^{2}$ \\ ${ }^{1}$ Department of Paediatrics, University Medical Centre Maribor, Maribor, Slovenia \\ ${ }^{2}$ Faculty of Medicine, University of Maribor, Maribor, Slovenia
}

Adv Dermatol Allergol 2022; XXXIX (4): 697-703

DOI: https://doi.org/10.5114/ada.2021.108426

\begin{abstract}
Introduction: Atopic dermatitis (AD) is a chronic pruritic inflammatory skin disease. It is associated with atopy, impaired skin barrier, skin infections and several other comorbidities.

Aim: To identify comorbidities and risk factors that influence the severity of AD in children.

Material and methods: We performed a cross-sectional study involving 52 children newly diagnosed with AD. The severity of AD was assessed with the SCORing Atopic Dermatitis (SCORAD) clinical tool. Levels of serum tryptase, zinc, selenium, and immunoglobulins A, G, M and E (IgA, IgG, IgM, and total IgE, respectively) were determined as well as allergen-specific $E$ antibodies (IgE) to the most common allergens. DNA samples from venous blood were screened for the most common mutations in the filaggrin gene.

Results: The median age of patients was 30 months. The median SCORAD index in patients with atopy was 47.8, compared to 27.2 in non-atopic patients $(p<0.01)$. We also found a significantly higher median SCORAD of 61.2 in patients with low serum IgM levels compared to 34.9 in patients with normal serum IgM levels $(p=0.03)$. A history of impetigo was also associated with a higher median SCORAD of 56.2 compared to 34.0 in patients without impetigo $(p=0.01)$.

Conclusions: Patients with $\mathrm{AD}$ and sensitisation to common allergens, low levels of IgM or a history of impetigo are at risk for more severe disease and, therefore, need more attention, meticulous skin care, proactive management and treatment of comorbidities, when possible.
\end{abstract}

Key words: atopic dermatitis, children, comorbidities, severity.

\section{Introduction}

Atopic dermatitis (AD) is a chronic inflammatory skin disease characterized by intense itching and eczema [1]. It usually begins in childhood and affects approximately $20 \%$ of children and $10 \%$ of adults in the developed world [2, 3].

$A D$ has a diverse clinical presentation, which depends on age. The diagnosis of AD is based mostly on the characteristic clinical picture [3, 4].

Although the pathogenesis of AD is not fully understood, available evidence suggests that disease results from a complex interaction between genetic and environmental factors resulting in impaired skin barrier function, immune dysregulation and skin infections [5].

A history of $A D$ in parents is the most important risk factor for the development of disease in children [6]. At least in some cases, loss-of-function mutations in the fil- aggrin gene are responsible for the transmission of the disease to offspring. Filaggrin (FLG) is an essential structural protein responsible for the regulation of epidermal homeostasis and integrity of the skin barrier [7]. Approximately $20 \%$ of children with mild to moderate AD have at least one mutant allele of the FLG gene [2].

The role of allergy in the pathogenesis of $A D$ is controversial. Patients with $A D$ are prone to produce specific immunoglobulins of E class (IgE) to common allergens (atopy). AD also represents an independent risk factor for the development of asthma and allergic rhinitis. However, only approximately one-third of children with $A D$ are actually allergic to food or air-borne allergens, and allergies are even less common in adolescents and adults with AD [2]. Sensitisation to food (cow milk, egg white, nuts) prevails in infants and toddlers, whereas sensitisation to airborne allergens (house dust mites, pets, pollen) becomes more com-

Address for correspondence: Assist. Prof. Vojko Berce MD, PhD, Department of Paediatrics, University Medical Centre Maribor, Ljubljanska 5, 2000 Maribor, Slovenia, phone: +386 2321 21 30, mobile phone: +386 31870 834, fax: +386 2331 45 31, e-mail: vojko.berce@guest.arnes.si Received: 29.04.2021, accepted: 29.05.2021. 
mon in older children. One-quarter of children with $A D$ are sensitized to more than five food and/or airborne allergens (polysensitisation), and those patients have a more severe form of the disease [8]. Immediate types of hypersensitivity with histamine release and cytokines produced by type 2 T-helper cells, which stimulate immunoglobulin (Ig) E synthesis, are detected in most patients with AD. Although hypersensitivity reactions enhance skin barrier dysfunction, they are a consequence and not a cause of $A D$ as an impaired skin barrier facilitates penetration of common allergens and sensitisation [5]. Not all patients with $A D$ develop IgE-mediated hypersensitivity to common food and/or airborne allergens; therefore, the term "atopic dermatitis" is rather misleading and is often substituted with "eczema" to encompass the atopic and non-atopic form of the disease [3].

$A D$ is associated with several other comorbidities and underlying conditions. It is frequently reported in children with (transient) hypogammaglobulinemia of infancy, and the disease resolves with the normalization of immunoglobulin $G$ (IgG) levels [9]. Children with AD were found to have a lower serum level of selenium and/or zinc than healthy children [10]. The level of serum tryptase was also found to be positively correlated with the severity of $A D$ in children [11]. Patients with $A D$ are prone to skin infections, and their skin is colonized with Staphylococcus aureus in $90 \%$ of cases. S. aureus and Malassezia spp. are probably involved in the stimulation of the immune system and upregulation of inflammation in patients with AD. Impetigo caused by S. aureus or Streptococcus pyogenes is a common complication of $\operatorname{AD}[3,12]$. Primary infection with herpes simplex virus occurs in the disseminated form (herpetic eczema) in 3\% of patients with $A D$ and is more prevalent in the severe and allergic form of AD. The severe form of AD is also associated with a widespread Molluscum contagiosum viral infection [13].

Quality of life is severely affected in patients with $A D$, mostly because of itching and impaired sleeping. In addition, the impact of disease on the physical appearance and social interactions is especially important in adolescence $[5,14]$. SCORing Atopic Dermatitis (SCORAD) is a clinical tool used to assess the extent and severity of the disease. The extent of the disease is assessed by the percentage of the affected body area and the severity, which is determined by scoring the intensity of 6 clinical characteristics (redness, swelling, oozing/crusting, scratch marks, lichenification and dryness of the skin) [15].

\section{Aim}

We aimed to determine which comorbidities and underlying conditions are associated with a more severe course of $A D$ to identify patients who need more attention and intensive management of $A D$ and of the comorbidities themselves.

We hypothesized that the following characteristics of patients with $A D$ are associated with a more severe form of the disease: family history of $A D$, young age, shorter duration of breastfeeding, history of asthma/wheezing or anaphylaxis, sensitisation (especially polysensitisation and sensitisation to nuts), hypogammaglobulinemia, increased serum levels of total IgE and tryptase, low serum levels of zinc and/or selenium, mutations in the FLG gene and skin infections.

\section{Material and methods}

\section{Participants}

We performed a cross-sectional study and included all newly diagnosed patients with $A D$ aged from 3 months to 18 years who were referred to the paediatric allergy outpatient clinic at our Division of Paediatrics from 1 October 2019 to 31 January 2021. The diagnosis of AD was established according to the criteria set by Hanifin and Rajka and criteria proposed by Williams (adapted from the original United Kingdom working party's diagnostic criteria for atopic dermatitis) $[16,17]$. We excluded patients with other chronic skin diseases, such as psoriasis, allergic and irritant contact dermatitis, nummular eczema, seborrheic dermatitis, and children with congenital immune deficiencies or autoimmune or metabolic skin conditions. We did not exclude patients who were already receiving primary health care. However, patients included in the study should not be treated with any systemic or topical anti-inflammatory medications for at least 2 weeks before enrolment. We also did not exclude patients with other atopic diseases, such as asthma, allergic rhinitis and allergic diseases of the gastrointestinal tract.

The extent and severity of AD was independently assessed by two paediatric allergologists with the SCORAD clinical tool [15], and the mean of both scores was used for further analysis. We recorded the duration of breastfeeding and the presence (or history) of comorbidities such as asthma or virus-induced wheezing (at least two episodes in the past year) and anaphylaxis. We also inquired about the history of skin infections such as: herpetic eczema, impetigo and molluscum contagiosum.

\section{Laboratory investigations}

A venous blood sample was drawn from all patients for the analysis of complete and differential blood count. Levels of serum tryptase, zinc, selenium, and immunoglobulins A, G, $M$ and $E$ (IgA, IgG, IgM, total lgE) were determined. Specific IgE levels to house dust mite, cow's milk, egg white, wheat, cod fish, soya bean, peanuts and tree nuts were determined in all patients with ImmunoCAP assay (Phadia, Inc., Uppsala, Sweden). Specific IgE to other allergens (e.g. pets, moulds, pollens, other food) was determined according to the history of exposure or observed allergic reactions. A cut-off value of $0.35 \mathrm{kU} / \mathrm{l}$ was used to confirm allergic sensitisation [18].

DNA samples from venous blood were screened for the five most common loss-of-function mutations in the FLG gene [19].

All blood tests were performed in the central laboratory of the University Medical Centre Maribor, Slovenia, except for the determination of mutations in the FLG 
gene, which was performed in the Laboratory for Clinical Immunology and Molecular Genetics, University Clinic of Pulmonary and Allergic Diseases, Golnik, Slovenia.

\section{Ethical approval}

The study was approved by the Ethics Committee of the University Medical Centre Maribor, Slovenia and was performed according to the Declaration of Helsinki of 1975, revised in Edinburgh in 2000. All participants or their legal guardians (for children younger than 16 years) signed an informed consent form.

\section{Statistical analysis}

Statistical analysis was performed with IBM SPSS 24.0 software (IBM Inc., Chicago, IL, USA). The influence of qualitative variables (e.g., history of asthma/wheezing or anaphylaxis, presence of sensitisation, skin infections, FLG gene mutations, hypogammaglobulinemia, low serum levels of zinc and selenium) on the SCORAD index was analysed with the Mann-Whitney U-test after

Table 1. Qualitative epidemiological, clinical and laboratory characteristics of children with atopic dermatitis

\begin{tabular}{|c|c|c|}
\hline Characteristic & Frequency $(N)$ & Percentage \\
\hline Female sex & 22 & 42.3 \\
\hline Family history of AD & 26 & 50.0 \\
\hline Sensitization*: & 34 & 65.4 \\
\hline To nuts & 16 & 30.8 \\
\hline To $>5$ allergens & 13 & 25.0 \\
\hline Anaphylaxis & 1 & 1.9 \\
\hline Asthma/wheezing & 7 & 13.5 \\
\hline Increased serum total IgE levels** & 32 & 62.7 \\
\hline $\begin{array}{l}\text { Decreased serum immunoglobulin } \\
\text { levels }{ }^{* * *} \text { : }\end{array}$ & 11 & 21.2 \\
\hline $\lg A$ & 4 & 7.7 \\
\hline $\lg M$ & 8 & 15.4 \\
\hline $\lg G$ & 3 & 5.8 \\
\hline Increased serum tryptase levels & 2 & 3.8 \\
\hline Low serum selenium levels ${ }^{\star \star \star \star}$ & 18 & 38.3 \\
\hline Low serum zinc levels ${ }^{\star * * *}$ & 4 & 8.5 \\
\hline Filaggrin mutation & 8 & 15.6 \\
\hline Skin infections: & 11 & 21.2 \\
\hline Impetigo & 8 & 15.4 \\
\hline \multicolumn{3}{|c|}{$\begin{array}{l}\text { "Sensitization to common food and/or airborne allergens. " Serum levels of } \\
\text { total immunoglobulin E above the upper normal range for age [21]. }{ }^{* * *} \text { Serum } \\
\text { levels of immunoglobulin classes } A, G \text { and } M \text { below the lower normal range } \\
\text { for age [20]. } \text { Serum levels of selenium and/or zinc below the lower normal } \\
\text { range for age [22]. }\end{array}$} \\
\hline
\end{tabular}

Table 2. Quantitative epidemiological, clinical and laboratory characteristic of children with atopic dermatitis

\begin{tabular}{|c|c|c|}
\hline Characteristic & Median & $\begin{array}{c}\text { Interquartile } \\
\text { range }\end{array}$ \\
\hline Age [months] & 30 & 84 \\
\hline Duration of breastfeeding [months] & 6 & 6 \\
\hline SCORAD index & 41.3 & 30.8 \\
\hline Serum total lgE levels [IU/ml] & 79 & 312 \\
\hline Normalized $(\%)^{\star}$ & 240 & 996 \\
\hline Serum IgA levels $[\mathrm{g} / \mathrm{l}]$ & 0.58 & 0.86 \\
\hline Normalized $(\%)^{\star *}$ & 248 & 255 \\
\hline Serum IgM levels $[\mathrm{g} / \mathrm{l}]$ & 0.68 & 0.54 \\
\hline Normalized (\%) ${ }^{\star \star}$ & 157 & 114 \\
\hline Serum IgG levels [g/l] & 7.13 & 5.93 \\
\hline Normalized (\%)** & 164 & 63 \\
\hline Serum tryptase levels [ng/ml] & 4.64 & 2.43 \\
\hline Serum selenium levels $[\mu \mathrm{mol} / \mathrm{l}]$ & 71.2 & 19.1 \\
\hline Normalized $(\%)^{\star * *}$ & 110 & 33 \\
\hline Serum zinc levels $[\mu \mathrm{mol} / \mathrm{l}]$ & 14.5 & 5.4 \\
\hline Normalized $(\%)^{\star \star \star}$ & 132 & 42 \\
\hline
\end{tabular}

*Serum levels of total immunoglobulin E expressed as a percentage of the upper normal range for age [21]. **Serum levels of immunoglobulin classes $A, G$ and $M$ expressed as a percentage of the lower normal range for age [20]. ${ }^{* * *}$ Serum levels of selenium and zinc expressed as a percentage of the lower normal range for age [22]. 


\section{Influence of characteristics and comorbidities on the severity of $A D$}

The influence of quantitative epidemiological, clinical and laboratory characteristics on the severity of atopic dermatitis (expressed as the SCORAD index) is presented in Table 3.

The correlation of the severity of AD (expressed as the SCORAD index) with quantitative epidemiological, clinical and laboratory characteristics is presented in Table 4.

When testing with a multiple linear regression model, we found that the association of the severity of $A D$ (SCORAD index) and sensitisation (to common food and/ or airborne allergens) remained significant $(p<0.01)$, and the association with the history of impetigo was marginally significant $(p=0.05)$.

In addition, we performed a statistical analysis separately for young children (0-36 months of age, $N=27$ ) and older children (> 36 months, $N=25$ ). In young children only the history of impetigo was found to be significantly associated with severity of atopic dermatitis (median SCORAD of 56.3, compared with 33.5 in those without impetigo, $p=0.01)$. In this age group, we also observed a positive correlation between SCORAD index and normalized total lgE serum levels ( $\rho=0.43, p=0.03$ ). In children older than 36 months, we found a significant association with more severe AD for the presence of sensitisation to common allergens (median SCORAD index of 51.2 compared to 24.9 in those without sensitisations, $p=0.01$ ), hypogammaglobulinemia M (median SCORAD index of 70.9, compared to 34.9 in those without hypogammaglobulinemia $M, p=0.01$ ) and the history of impetigo (median SCORAD index of 64.4, compared to 34.0 in those without impetigo, $p<0.01$ ).

\section{Discussion}

In our cross-sectional study, we found that sensitisation to food and/or airborne allergens, low levels of serum IgM and a history of impetigo were associated with a more severe form of $A D$. The severity of $A D$ (expressed as the SCORAD index) was positively correlated with the serum levels of total IgE.

To date, several studies have documented an increased risk of allergies in patients with $A D$, especially in preschool age. However, sensitisation to common food and/or airborne allergens is probably a consequence of an impaired skin barrier in patients with $A D$ and not a cause of the disease, and most sensitized patients do not have any symptoms or worsening of AD when exposed to those allergens [3]. Similar to our results, Hon et al. and Kuo et al. found a positive correlation between the SCORAD index and serum levels of total IgE in Chinese and Taiwanese children with $A D$, respectively. In addition, Kuo et al. reported a correlation between the number of allergens to which the patient was sensitized and

Table 3. Influence of qualitative characteristics and comorbidities on SCORAD index in children with atopic dermatitis

\begin{tabular}{|c|c|c|c|}
\hline Characteristic & Yes* [median (IQR)] & $\mathrm{No}^{\star}$ [median (IQR)] & $P$-value \\
\hline Female sex & $34.1(36.2)$ & $44.2(28.9)$ & 0.73 \\
\hline Family history of $A D$ & $49.3(26.5)$ & $34.9(32.8)$ & 0.19 \\
\hline Sensitization: & $47.8(27.5)$ & $27.2(32.3)$ & $<0.01$ \\
\hline To nuts & $41.3(23.0)$ & $54.1(34.6)$ & 0.60 \\
\hline To $>5$ allergens & $56.6(33.6)$ & $45.5(28.0)$ & 0.28 \\
\hline Asthma/wheezing & $54.7(35.1)$ & $39.6(32.1)$ & 0.37 \\
\hline Increased serum total IgE levels** & $44.2(32.1)$ & $33.8(33.9)$ & 0.25 \\
\hline Decreased serum immunoglobulin levels ${ }^{\star \star *}$ : & $58.2(33.6)$ & $37.6(31.2)$ & 0.30 \\
\hline $\lg A$ & $33.2(24.4)$ & $44.2(31.2)$ & 0.46 \\
\hline $\lg M$ & $61.2(31.6)$ & $34.9(29.6)$ & 0.03 \\
\hline $\lg G$ & $19.8(30.0)$ & $43.0(30.1)$ & 0.24 \\
\hline Low serum selenium levels**** & $39.3(40.3)$ & $34.2(28.7)$ & 0.95 \\
\hline Low serum zinc levels ${ }^{\star \star \star \star}$ & $34.9(42.4)$ & $34.9(33.0)$ & 1 \\
\hline Filaggrin mutation & $55.8(26.5)$ & $34.2(32.1)$ & 0.08 \\
\hline Skin infections: & $55.9(36.9)$ & $33.8(30.9)$ & 0.05 \\
\hline Impetigo & $56.2(20.9)$ & $34.0(32.5)$ & 0.01 \\
\hline
\end{tabular}

*Presence (YES) or absence (NO) of certain characteristic and comparison of SCORAD index between those two groups. **Serum levels of total immunoglobulin E expressed as a percentage of the upper normal range for age [21]. ${ }^{* * *}$ Serum levels of immunoglobulin classes $A, G$ and $M$ expressed as a percentage of the lower normal range for age [20]. ${ }^{* * *}$ Serum levels of selenium and zinc expressed as a percentage of the lower normal range for age [22]. 
the severity of the disease $[23,24]$. The correlation of the SCORAD index and the number of sensitisations in young children with AD was also reported by Wahn et al. [25]. However, in our study, polysensitisation (sensitisation to five or more allergens) was not associated with the severity of AD. We found a polysensitisation in $25 \%$ of our patients, which is similar to that reported by Broeks and Brand, who found polysensitisation in $22.4 \%$ of children with $A D$ [8]. Similar to our findings, Flohr et al. reported a strong association between AD severity and sensitisation to food allergens in infants, which was independent of transepidermal water loss and FLG mutations [26]. These and our results (lack of influence of polysensitisation on the severity of $A D$ ), therefore, only partially support the hypothesis that impaired skin barrier in patients with AD facilitates sensitisation to common food and/or airborne allergens [27]. We detected a sensitisation to one or more common food and/or airborne allergens in almost two-thirds of our patients, which is even more than $30-60 \%$ as reported previously [28]. However, only 1 (1.9\%) of our patients reported anaphylaxis, which further supports data about the discordance between a high prevalence of sensitisation and a low actual rate of clinical allergy in patients with AD [29].

We observed a relatively high prevalence (30.8\%) of sensitisation to nuts (peanuts and/or tree nuts) in our patients, although only 1 (1.9\%) of them actually reported a serious allergic reaction. Therefore, we can assume that the association of the presence of allergen-specific IgE and the correlation of total IgE with disease severity in our study both reflect dysregulation of the immune system in patients with $A D$, rather than sensitisation to common food and/or airborne allergens because of a dysfunctional skin barrier. High-risk sensitisation would probably result in a higher rate of serious allergic reactions to nuts as Sicherer et al. reported a 50\% incidence of life-threatening reactions at ingestion among children allergic to nuts and/or peanuts [30].

Asthma or viral-induced wheezing was reported in $7(13.5 \%)$ of our patients, which is not significantly higher than the $8-10 \%$ prevalence of asthma in the general population of children in developed countries [31] and is much lower than the 30-39\% reported by Wan et al. in children with $\mathrm{AD}$ [32]. The lower prevalence of asthma can be partly explained by the lower median age (30 months) of our patients as in this age group, the diagnosis of asthma is frequently underestimated [33]. In contrast to allergic asthma, the association of viral-induced wheezing with $A D$ is not well known, although mutations in the FLG gene predispose patients to viral respiratory illnesses and wheezing [34]. However, our results do not support the association of virus-induced wheezing and AD.

We found that patients with serum IgM levels below the age-adjusted lower normal range [20] had a very severe form of the disease, with a median SCORAD index of 61.2. Patients with primary immune deficiencies,
Table 4. Correlation of quantitative epidemiological and laboratory characteristics with SCORAD index in children with atopic dermatitis

\begin{tabular}{|c|c|c|}
\hline Characteristic & $\begin{array}{l}\text { Correlation } \\
\text { coefficient }^{*}\end{array}$ & $P$-value \\
\hline Age & -0.05 & 0.73 \\
\hline Duration of breastfeeding & -0.08 & 0.59 \\
\hline Serum total lgE levels (normalized) ${ }^{\star *}$ & 0.35 & 0.01 \\
\hline Serum IgA levels (normalized) ${ }^{\star \star \star}$ & -0.01 & 0.96 \\
\hline Serum IgM levels (normalized) ${ }^{\star \star \star}$ & -0.12 & 0.40 \\
\hline Serum IgG levels (normalized) ${ }^{\star \star \star}$ & 0.06 & 0.65 \\
\hline Serum tryptase levels & 0.26 & 0.09 \\
\hline Serum selenium levels (normalized) ${ }^{\star \star \star \star}$ & -0.17 & 0.25 \\
\hline Serum zinc levels (normalized) ${ }^{\star * * *}$ & 0.16 & 0.30 \\
\hline
\end{tabular}

*Spearman's rank-order correlation coefficient. **Serum levels of total immunoglobulin E expressed as a percentage of the upper normal range for age [21]. ***Serum levels of immunoglobulin classes $A, G$ and $M$ expressed as a percentage of the lower normal range for age [20]. ${ }^{* * *}$ Serum levels of selenium and zinc expressed as a percentage of the lower normal range for age [22].

such as Wiskott-Aldrich syndrome and severe combined immunodeficiency (SCID), often suffer from intractable eczema, which is similar to the severe form of AD [35]. However, none of our patients had a primary immunodeficiency as such subjects were excluded from the study. Patients with transient hypogammaglobulinemia of infancy (THI) often express atopic manifestations with increased lgE levels, sensitisation to food and severe eczema. However, children with THI usually have lower serum levels of IgG, and isolated lower serum levels of IgM are rarely encountered in this entity $[9,36]$. In our study, lower levels of IgA and IgG were not associated with the more severe form of $A D$ and we observed an association of hypogammaglobulinemia $M$ with a more severe form of AD only in the subgroup of patients older than 36 months. Therefore, we cannot attribute our results to the presence of THI.

We also observed a significantly higher SCORAD index in patients with a history of impetigo. Skin infections with bacteria and viruses are much more common in patients with $A D$ than in the general population. Almost $90 \%$ of patients with AD are colonized with S. aureus, and children with $A D$ have an increased risk of impetigo due to the compromised barrier function and immune dysregulation [37]. S. aureus acts as a superantigen and non-specifically upregulates the immune response and exacerbates skin inflammation [13]. We did not observe any association of other skin infections (such as herpetic eczema) with disease severity, which can be attributed to the low number of such patients in our cohort as only two of them had a positive history of herpetic eczema.

We detected at least one loss-of-function mutation in the FLG gene in 8 (15.6\%) patients with AD. However, our patients with a detected mutation did not have a signifi- 
cantly higher SCORAD index. Margolis et al. reported that patients with AD who carry at least one copy of R501X or 2282del4 mutations in the FLG gene have a fourfold higher risk of AD. They also found a prevalence of at least one loss-of-function mutation in $31.5 \%$ of white children with AD, was higher than that in our cohort. However, Margolis et al. analysed the presence of approximately 500 loss-offunction mutations, which is far more than was analysed in our study [38]. We observed a median SCORAD index of 55.8 in patients with detected mutations and 34.2 in patients without mutations. However, this difference was not found to be significant ( $p=0.08$ ), which can be attributed to the relatively small number of patients with mutations who were included in our study.

Lower serum levels of zinc and selenium were not associated with the severity of AD in our study. Serum levels of both microelements were found to be lower in children with $A D$ than in healthy controls in previous studies and meta-analyses [10]. Therefore, low serum levels of zinc and/or selenium may be involved in the pathogenesis of AD but do not contribute to the severity of disease.

We did not confirm a correlation between the serum tryptase levels and SCORAD index as was reported previously by Sahiner et al. in infants and toddlers with AD. In addition, Sahiner et al. reported significantly higher serum tryptase levels (median $5.01 \mathrm{ng} / \mathrm{ml}$ ) in sensitised children with $A D$ than in children with the non-atopic form of AD (median $3.02 \mathrm{ng} / \mathrm{ml}$ ) [11]. In our cohort, there was no difference in the serum tryptase levels between the sensitised (median value of $4.64 \mathrm{ng} / \mathrm{ml}$ ) and nonsensitised (median value of $4.68 \mathrm{ng} / \mathrm{ml}$ ) patients with AD (not shown in the Results section). Our results do not suggest that serum tryptase levels could represent a sensitive biomarker in AD.

Family history of AD was not found to influence the severity of the disease in our study. Otherwise, the family history of allergic diseases and especially of $A D$ is the single most important risk factor for the development of $A D$ with a three- to five-fold increased risk of $A D$ in offspring, when one or both parents have $A D$, respectively [6]. However, risk factors for the disease itself and its severity often differ and our results regarding the family history and severity of $A D$ probably reflect the lack of influence of FLG mutations on the severity of $A D$ in our patients.

One of the major limitations of our study is a relatively low number of participants, which is a consequence of the inclusion criteria as only newly diagnosed and untreated (at least recently) patients were enrolled. Second, most of our patients had a moderate or severe form of AD (median SCORAD index was 41.3) as patients with a mild form are usually not referred to our outpatient clinic. Therefore, the findings of our study cannot be applied to patients with mild AD. Third, microbiological diagnostic tests were not performed in most of our patients with impetigo and therefore we could not analyse the influence of different types of impetigo (e.g., streptococcal, staphylococcal, etc.) on the severity of AD.

\section{Conclusions}

Children with AD may suffer from several comorbidities. Some of them are associated with the pathogenesis of the disease, and others are the consequence of AD. Examples of such comorbidities are hypogammaglobulinemia, sensitisation to common food and/or airborne allergens and bacterial skin infections. We found that the presence of those comorbidities in patients with $A D$ was associated with a more severe form of the disease. Therefore, the identification and treatment of such comorbidities should represent an important part of the management of children with AD.

\section{Acknowledgments}

We would like to thank our colleagues Maja Tomazin, MD, Brigita Koren, MD and Tina Hojnik, MD from our Division of Paediatrics for including patients who were treated by them and Urška Bidovec Stojkovič from the Laboratory for Clinical Immunology and Molecular Genetics, University Clinic of Pulmonary and Allergic Diseases, Golnik, Slovenia for performing a genetic analysis.

Study was conducted in: University Medical Centre Maribor

Attributed to: Department of Paediatrics, University Medical Centre Maribor.

\section{Conflict of interest}

The authors declare no conflict of interest.

\section{References}

1. Eichenfield LF, Tom WL, Chamlin SL, et al. Guidelines of care for the management of atopic dermatitis: section 1. Diagnosis and assessment of atopic dermatitis Work Group. J Am Acad Dermatol 2014; 70: 338-51.

2. Weidinger S, Beck LA, Bieber T, et al. Atopic dermatitis. Nat Rev Dis Primers 2018; 4: 1.

3. Langan SM, Irvine AD, Weidinger S. Atopic dermatitis. Lancet 2020; 396: 345-60.

4. Yew YW, Thyssen JP, Silverberg JI. A systematic review and meta-analysis of the regional and age-related differences in atopic dermatitis clinical characteristics. J Am Acad Dermatol 2019; 80: 390-401.

5. Kapur S, Watson W, Carr S. Atopic dermatitis. Allergy Asthma Clin Immunol 2018; 14 (Suppl 2): 52.

6. Ravn NH, Halling AS, Berkowitz AG, et al. How does parental history of atopic disease predict the risk of atopic dermatitis in a child? A systematic review and meta-analysis. J Allergy Clin Immunol 2020; 145: 1182-93.

7. van den Oord RAHM, Sheikh A. Filaggrin gene defects and risk of developing allergic sensitisation and allergic disorders: systematic review and meta-analysis. BMJ 2009; 339: b2433. 
8. Broeks SA, Brand PLP. Atopic dermatitis is associated with a fivefold increased risk of polysensitisation in children. Acta Paediatr 2017; 106: 485-8.

9. Sumikawa Y, Kato J, Kan Y, et al. Severe atopic dermatitis associated with transient hypogammaglobulinemia of infancy. Int J Dermatol 2015; 54: 185-7.

10. Vaughn AR, Foolad N, Maarouf M, et al. Micronutrients in atopic dermatitis: a systematic review. J Altern Complement Med 2019; 25: 567-77.

11. Sahiner UM, Buyuktiryaki B, Gungor HE, et al. Factors that predict disease severity in atopic dermatitis: the role of serum basal tryptase. Allergy Asthma Proc 2018; 39: 371-6.

12. Nowicka D, Nawrot U. Contribution of Malassezia spp. to the development of atopic dermatitis. Mycoses 2019; 62: 588-96.

13. Fenner J, Silverberg NB. Skin diseases associated with atopic dermatitis. Clin Dermatol 2018; 36: 631-40.

14. Drucker AM, Wang AR, Li WQ, et al. The burden of atopic dermatitis: summary of a report for the National Eczema Association. J Invest Dermatol 2017; 137: 26-30.

15. Stalder JF, Taïeb A, Atherton DJ, et al. Severity scoring of atopic dermatitis: the SCORAD index: consensus report of the European task force on atopic dermatitis. Dermatology 1993; 186: 23-31.

16. Hanifin, Rajka G. Diagnostic features of atopic dermatitis. Acta Derm Venereol (Stockh) Suppl 1980; 92: 44-7.

17. Williams HC. Atopic dermatitis. N Engl J Med 2005; 352: 2314-24

18. Wood RA, Segall N, Ahlstedt S, Williams PB. Accuracy of IgE antibody laboratory results. Ann Allergy Asthma Immunol 2007; 99: 34-41.

19. Sandilands A, Terron-Kwiatkowski A, Hull PR, et al. Comprehensive analysis of the gene encoding filaggrin uncovers prevalent and rare mutations in ichthyosis vulgaris and atopic eczema. Nat Genet 2007; 39: 650-4.

20. Jolliff CR, Cost KM, Stivrins PC, et al. Reference intervals for serum IgG, IgA, IgM, C3, and C4 as determined by rate nephelometry. Clin Chem 1982; 28: 126-8.

21. Kjellman NM, Johansson SG, Roth A. Serum IgE levels in healthy children quantified by a sandwich technique (PRIST). Clin Allergy 1976; 6: 51-9.

22. Rükgauer M, Klein J, Kruse-Jarres JD. Reference values for the trace elements copper, manganese, selenium, and zinc in the serum/plasma of children, adolescents, and adults. J Trace Elem Med Biol 1997; 11: 92-8.

23. Hon KLE, Lam MCA, Leung TF, et al. Are age-specific high serum IgE levels associated with worse symptomatology in children with atopic dermatitis? Int J Dermatol 2007; 46: 1258-62.

24. Kuo HC, Chu CH, Su YJ, Lee CH. Atopic dermatitis in Taiwanese children: the laboratory values that correlate best to the SCORAD index are total IgE and positive Cheddar cheese lgE. Medicine 2020; 99: e21255.

25. Wahn U, Warner J, Simons FER, et al. IgE antibody responses in young children with atopic dermatitis. Pediatr Allergy Immunol 2008; 19: 332-6.

26. Flohr C, Perkin M, Logan K, et al. Atopic dermatitis and disease severity are the main risk factors for food sensitization in exclusively breastfed infants. I Invest Dermatol 2014; 134: 345-50.

27. Lowe AJ, Leung DYM, Tang MLK, et al. The skin as a target for prevention of the atopic march. Ann Allergy Asthma Immunol 2018; 120: 145-51.
28. Eller E, Kjaer HF, Høst A, et al. Food allergy and food sensitization in early childhood: results from the DARC cohort. Allergy 2009; 64: 1023-9.

29. Spergel JM, Boguniewicz M, Schneider L, et al. Food allergy in infants with atopic dermatitis: limitations of food-specific IgE measurements. Pediatrics 2015; 136: 1530-8.

30. Sicherer SH, Burks AW, Sampson HA. Clinical features of acute allergic reactions to peanut and tree nuts in children. Pediatrics 1998; 102: e6.

31. Anandan C, Nurmatov U, Van Schayck OCP, Sheikh A. Is the prevalence of asthma declining? Systematic review of epidemiological studies. Allergy 2010; 65: 152-67.

32. Wan J, Mitra N, Hoffstad OJ, et al. Variations in risk of asthma and seasonal allergies between early- and late-onset pediatric atopic dermatitis: a cohort study. J Am Acad Dermatol 2017; 77: 634-40.

33. Bisgaard H, Bonnelykke K. Long-term studies of the natural history of asthma in childhood. J Allergy Clin Immunol 2010; 126: 187-97.

34. Henderson J, Northstone K, Lee SP, et al. The burden of disease associated with filaggrin mutations: a populationbased, longitudinal birth cohort study. J Allergy Clin Immunol 2008; 121: 872-7.

35. Navabi B, Upton JEM. Primary immunodeficiencies associated with eosinophilia. Allergy Asthma Clin Immunol 2016; 12: 27.

36. Kidon MI, Handzel ZT, Schwartz R, et al. Symptomatic hypogammaglobulinemia in infancy and childhood - clinical outcome and in vitro immune responses. BMC Fam Pract 2004; 5: 23.

37. Salah LA, Faergemann J. A retrospective analysis of skin bacterial colonisation, susceptibility and resistance in atopic dermatitis and impetigo patients. Acta Derm Venereol 2015; 95: 532-5.

38. Margolis DJ, Mitra N, Wubbenhorst B, et al. Association of filaggrin loss-of-function variants with race in children with atopic dermatitis. JAMA Dermatol 2019; 155: 1269-76. 